\title{
Un notable antecedente del enfoque comunicativo en la enseñanza de lenguas extranjeras y de la apertura europeísta en la España de principios del siglo XX: Memoria histórica de la enseñanza de idiomas
}

\author{
MAR ViÑa Rouco \\ Observatorio Atrium Linguarum para las LES \\ Universidad de Santiago de Compostela
}

Recibido: 19 noviembre 2007 / Aceptado: 31 marzo 2008

ISSN: 1697-7467

\begin{abstract}
RESUMEN: En este artículo nos referimos a un movimiento de renovación pedagógica en la enseñanza - aprendizaje de lenguas extranjeras en España en el primer tercio del siglo XX que podría ser considerado como un claro precedente de la metodología comunicativa. Dentro del ámbito de la Institución Libre de Enseñanza José Castillejo y Duarte proclamó la necesidad del aprendizaje de varios idiomas modernos y de una educación internacional. Una de sus creaciones más acertadas, la Escuela Internacional Española, sirve de laboratorio para la puesta en práctica de estas ideas innovadoras. La pregunta que nos hacemos es qué hubiese ocurrido en cuanto a la innovación educativa en idiomas si esta floreciente actividad no hubiese acabado abruptamente con la Guerra Civil.

Palabras clave: Precedente de la metodología comunicativa; Institución Libre de Enseñanza; Escuela Internacional Española; educación plurilingüe a principios del siglo XX; método natural.
\end{abstract}

A notorious antecedent of the communicative approach in foreign language teaching and the European opening in the early $20^{\text {th }}$ century Spain: a historical memory of language teaching

ABSTRACT: In this paper we refer to an innovative pedagogic movement in the teaching and learning of foreign languages in Spain at the beginning of the $20^{\text {th }}$ century. It might be considered as a clear precedent of the communicative approach. Within the Institución Libre de Enseñanza, José Castillejo y Duarte proclaimed the need to learn several foreign languages within an international educational context. He was the founder of the Spanish International School, in which these innovative ideas were put into practice. It is wondered what would have happened if this interesting experiment hadn't been abruptly finished with the onset of the Spanish Civil War.

Key words: Communicative methodology precedent; the Free School; the Spanish International School; multilingual education in the early $20^{\text {th }}$ Century; Natural Method. 


\section{InTRODUCCIÓN}

Si bien a lo largo del siglo XIX y gran parte del XX se ha observado que en España hubo un claro predominio del paradigma tradicional en la enseñanza-aprendizaje de los idiomas modernos, no podemos dejar de mencionar la existencia de un movimiento de renovación pedagógica en general, y en la enseñanza de las lenguas vivas en particular, que comienza (del mismo modo que el Movimiento Reformista liderado por Wilhelm Viëtor) a finales del siglo XIX y se prolonga durante el primer tercio del siglo XX, acabando bruscamente con el inicio de la Guerra Civil y que bien podría considerarse como precursor de la enseñanza comunicativa actual.

Esta renovación en dicha enseñanza-aprendizaje sólo llega a ser experimentada por la élite intelectual de la época y aparece fuertemente ligada a la Institución Libre de Enseñanza. Sobre ésta última se han publicado numerosos e interesantes trabajos. Fue fundada en 1876 por Francisco Giner de los Ríos, cuando éste y otros educadores fueron separados de sus cátedras por sus opiniones heterodoxas. ${ }^{1}$ A pesar de esto pudieron continuar dedicándose a la educación prescindiendo de la tutela gubernamental y eclesiástica. La libertad de pensamiento, el individualismo, la negación de la autoridad y el europeísmo fueron los pilares básicos de dicha institución. Este deseo de abrirse hacia Europa, en especial hacia lo anglosajón, hace que la enseñanza de las lenguas vivas ocupe un puesto destacado en el currículum escolar de la Institución. Francisco Giner de los Ríos siempre apoyó y alentó el intercambio cultural de alumnos y profesores con otros países europeos, especialmente con los anglosajones. Como señala (J.B. Trend 1921: 21-22):

The idea of excursiones escolares and exchange of students he got from Paris; he learned a great deal from the masterly way in which the Germans were then reorganizing their own system; but he was never tired of thanking and complimenting the English for the sympathy and good will which they had shown him and the very real interest which his English friends always took in his undertaking. Don Francisco insisted that his pupils should travel.

Según Trend, había algo típicamente inglés en el modo de trabajar de estos reformistas: un trabajo metódico, duro y silencioso, sin ningún tipo de propaganda. Si los ideales básicos eran los de una enseñanza libre, experimental, innovadora y creativa no es de extrañar que la metodología de la enseñanza-aprendizaje de las lenguas vivas que más se favorecía en esta institución fuese la Metodología Directa ó Método Natural.

También asistiremos a un proceso de democratización de la enseñanza de idiomas modernos, los cuales paulatinamente se van ofertando a toda la sociedad en general. Esto no era así en épocas no tan lejanas. En este sentido José Castillejo (1929) señala en el periódico El Sol:

\footnotetext{
${ }^{1}$ «La Institución Libre de Enseñanza es completamente ajena a todo espíritu e interés de comunión religiosa, escuela filosófica o partido político; proclamando tan sólo el principio de la libertad e inviolabilidad de la ciencia y de la consiguiente independencia de su indagación y exposición respecto de cualquiera otra autoridad que la de la propia conciencia del profesor, único responsable de sus doctrinas.» (art. 15 de los estatutos de la I.L.E)
} 
La aspiración a dominar varias lenguas no es una novedad; pero ha sido hasta tiempo reciente un lujo o una necesidad de algunos centenares de personas... De lo que se trata precisamente es de extender ese beneficio más allá de aquellos privilegiados, poniéndolo al alcance de todo el mundo, sin exigir desplazamientos ni extraordinarios recursos...2

Asimismo, los problemas que aquejan al proceso de enseñanza - aprendizaje de idiomas modernos se repiten a lo largo de la historia, como, por ejemplo, el consenso unánime entre los especialistas de todas las épocas en cuanto al fracaso obtenido por un buen número de alumnos después de varios años de estudio de una lengua extranjera en la escuela. En este sentido, José Castillejo (1928) afirma que:

[es] sorprendente [el] fracaso de las escuelas secundarias que hasta en los países donde han aplicado insistencia y técnica admirables, no han obtenido sino resultados mediocres. En cuatro o cinco años dedicados a una lengua no consiguen que los alumnos la hablen ni la escriban, ni siquiera que la entiendan más que una reducida minoría. ${ }^{3}$

La perspectiva histórica nos permite realizar un seguimiento de ciertos aspectos y comprobar que las ideas consideradas como novedosas en la actualidad tienen claros antecedentes en el pasado, es decir, el germen de ciertos conceptos modernos ya existía aunque la teoría no estuviese explícitamente formulada. Así, hay indicios de la enseñanza comunicativa de la lengua en Europa desde el siglo XVI; a finales del siglo XIX, esta aproximación resurge con el Enfoque Natural, retomado recientemente por Krashen (1983). En nuestro país, sin embargo, esta ideología penetra más tarde, siendo accesible solamente a los intelectuales de finales del siglo XIX y principios del XX. Algunas ideas relevantes de Otto Jespersen aparecen traducidas y publicadas en el BILE ${ }^{4}$ (Boletín de la Institución Libre de Enseñanza) $n^{\circ} 788$, de 30 de noviembre de 1925 , en donde se afirma lo siguiente:

En cuanto a método, debe abandonarse por completo el artificial sustituyéndolo por otro más natural. Según el método artificial, lo primero que se hace es poner en manos del muchacho una gramática, para que se la trague toda... ¡Qué viejas son las quejas contra este sistema pervertido! ¡Cuántos suspiros no ha ocasionado, cuántas deformidades no ha producido! En cambio el método natural de aprender las lenguas es todo práctico. Es el mismo procedimiento por el que adquirimos nuestra lengua materna. (1925: 361)

La vocación europeísta de la Institución Libre de Enseñanza logra que entre 1901 y 1903 se otorguen las primeras becas para formarse en el extranjero. En 1907 se funda el organismo que iba a cambiar el panorama español a través de la educación: se aprueba por Real Decreto 5

\footnotetext{
${ }^{2}$ Vid. José Castillejo, «La Enseñanza Plurilingüe« El Sol 01-01-1929.

${ }^{3}$ Vid. José Castillejo, «La Enseñanza de las lenguas vivas» El Sol, 30-12-1928.

${ }^{4} \mathrm{El}$ artículo se titulaba «La enseñanza de las lenguas extranjeras» y reflejaba las principales ideas que aparecen en la obra de Otto Jespersen (1904) How to Teach a Foreign Language.

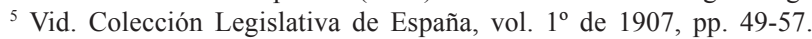


la constitución de la Junta para Ampliación de Estudios e Investigaciones Científicas (J.A.E), inspirada por Francisco Giner y por su discípulo que pasa a ser su secretario José Castillejo y Duarte. La labor de la Junta era poner a España en un nivel cultural comparable al de otros países europeos, es decir, transformar la educación española en todos sus aspectos. Esto se lograría a través de dos acciones básicas:

a) Provocar una corriente de comunicación científica y pedagógica con el extranjero

b) Agrupar en núcleos de trabajo intenso y desinteresado los elementos disponibles del país ${ }^{6}$

En primer lugar se intenta preparar adecuadamente a los futuros profesores (entre ellos, los de idiomas modernos) mediante becas en el extranjero. De este modo a su retorno, además de formar grupos de investigación, asumirían puestos docentes en institutos, escuelas normales y universidades. Para tener una idea del tipo de trabajo que realizaban los becarios interesados en el área de las lenguas vivas analizaremos brevemente algunas de las becas concedidas por la J.A.E. en el curso 1933-1934:

- Emilio Alemany Bolufer, director de la Escuela profesional de Comercio de Gijón. O.M. 26-08-1933. Tres meses en Alemania. Idioma: Alemán. El Sr. Alemany hizo un trabajo de sintaxis alemana, escrito en alemán, para que los alumnos se ejercitasen en la práctica del idioma y para que los del segundo curso pudiesen expresarse en él desde el primer día de clase.

- Bigot Armenta Moreno, catedrático de francés del instituto de Ceuta. O.M. 24-071933. Un mes en Francia. Ampliación de Francés y fonética. Las conferencias de fonética francesa se llevaron a cabo en el Instituto de Fonética de la Sorbona y estuvieron a cargo de M. Fouché, profesor de la Sorbona. Al final del curso los alumnos que quisieran someterse a examen recibieron el diploma correspondiente. El Sr. Armenta así lo hizo.

- Máximo Montes Lueje, Catedrático de la Escuela profesioal de Comercio de Alicante. O.M. 26-08-1933. Nueve meses en Inglaterra. Fonética Inglesa. Se matriculó en el Departamento de Fonética que dirigía en el University College de Londres el ilustre profesor Daniel Jones. Se matriculó en los siguientes cursos:

- Fonética del inglés

- Gramática del inglés hablado

- Elementos de la lingüística

- Lingǘstica general

- Principios de la Dicción

- Poesía inglesa antigua

- Defectos del lenguaje

- Eloísa González Sarriá, auxiliar de Alemán del Instituto de Idiomas de la Universidad de Valladolid. O.M. 10-05-1933. Seis meses en Alemania. Alemán.

- Dolores Sacristán Colás, maestra interina. O.M. 13-06-1934. Mes y medio en Inglaterra. Inglés y prácticas de organización escolar. ${ }^{7}$

\footnotetext{
${ }^{6}$ J.A.E. Memoria 1907.1909, p.4.

7 J.A.E. Memorias 1933-1934.
} 
Además de las pensiones, también existía un servicio de intercambio de becarios con varios países europeos y Estados Unidos. En el año 1933, y en lo que al intercambio con Estados Unidos se refiere, se concedieron ayudas para viajar a Dña. Lucinda Moles, designada por la Junta para disfrutar la beca ofrecida por el Wellesley College, y a Dña. Elisa Bernis, propuesta para la beca ofrecida por el Smith College. La Junta, a cambio, admitió como becarias a Ms. Janice Mackenzie y a Ms. Esther B. Aderson, procedentes del Wellesley College y del Smith College, respectivamente. En 1934 se concertaron dos intercambios con el Smith College, designando la Junta a Dña. María Antonia Sanjurjo y a Dña. Dolores Ibarra para las dos becas ofrecidas por dicho College, aceptando a cambio a las estudiantes norteamericanas Ms. Helen Mary Brooks y Ms. Francis Byrne.

La Junta a través del Patronato de estudiantes (creado por D.M de 09-06-1931) se ocupó, asimismo, de organizar el intercambio de niños y jóvenes con el extranjero, especialmente durante las vacaciones de verano. «De esta manera las familias españolas pueden facilitar a sus hijos el estudio de las lenguas y culturas de otros países, con un gasto que no grava su presupuesto sino en el coste de los viajes. El alumno español es recibido gratuitamente en una familia del país elegido, de condición análoga a la suya, y considerado como un miembro de aquélla durante el tiempo de su residencia. En correspondencia con esto la familia española se compromete a recibir en las mismas condiciones al muchacho o muchacha que la familia extranjera envíe en cambio.» ${ }^{8}$

Se gestionaron, además, colonias en el extranjero. De este modo, los alumnos del I-E (Instituto-Escuela) dependiente de la I.L.E residían durante un mes en el extranjero (Inglaterra, Francia y Alemania) y luego recibían al grupo de alumnos extranjeros en España. Se organizaron, asimismo, Clubes de inglés, francés y alemán. Sus miembros solían reunirse una vez al mes y «con ocasión de un té se charlaba, oía y comentaba una conferencia» ${ }^{9}$ en los idiomas objeto de estudio, potenciándose, además, la correspondencia escolar internacional, en lo posible con alumnos extranjeros «de las mismas inclinaciones y gusto de estudios» ${ }^{10}$

Como acabamos de comprobar eran muchas las actividades organizadas dentro del marco general de la ILE para promover el estudio de las lenguas y culturas extranjeras que no dejan de asombrar al lector actual por su vigencia y modernidad. Pasamos a referirnos a otra de las creaciones más originales del Secretario de la Junta, José Castillejo y Duarte.

\section{La Escuela Internacional Española y la enseñanza de los idiomas MODERNOS}

Dentro de una postura de apertura hacia Europa, Castillejo siempre proclamó la necesidad del aprendizaje de varios idiomas modernos y de una educación internacional. Como señala (Gamero Merino, 1987: 221):

Aunque casi toda la actividad de Castillejo se centró en la obra de la Junta para Ampliación de Estudios, no podemos olvidar la organización y el apoyo de otros proyectos al margen de este organismo. Uno de esos interesantes proyectos fue la Escuela Internacional Española (1928).

\footnotetext{
${ }^{8}$ Ibidem.

${ }^{9}$ Ibidem. P. 442.

${ }^{10}$ Ibidem.
} 
La semblanza que nos ofrece J.B. Trend de Castillejo es harto elocuente:

If one were asked to say who was the most representative figure of modern Spain, it would not be far wrong to name Sr. Castillejo. [...] His fluency in all European languages is astounding, and he has the power of turning himself on, as it were, with a stream of words which bring before you with extraordinary force and clearness what he wishes to convey. It might be said of him that he is more interested in the method of teaching ${ }^{11}$ than the subject taught. ${ }^{12}$

Más adelante analizaremos la Memoria y Programas de dicha Escuela y podremos comprobar a la luz de los conocimientos psicolingüísticos actuales la modernidad y relevancia de las ideas que la sustentaban. Así, encontraremos precedentes del enfoque centrado en el alumno, la enseñanza oral de la lengua extranjera, las actividades lúdicas relacionadas con la clase de idioma moderno, la dramatización, la simulación, la integración de los aspectos culturales en la enseñanza del idioma moderno y los intercambios escolares.

La Escuela Internacional Española fue fundada en Madrid en 1928, por una asociación de carácter cooperativo y sin ánimo de lucro. El presidente de esta asociación era Pedro Salinas, catedrático de la Universidad de Sevilla, la Vicepresidenta $1^{\circ}$ era María del Mar P. de Vega, Doctora en Medicina, el Vicepresidente $2^{\circ}$ era José Castillejo, catedrático de la Universidad de Madrid, el secretario era Fernando Salvador, arquitecto, y el tesorero Cándido Bolívar y Pieltain, catedrático de la Universidad de Madrid.

El propósito fundamental era el de educar a niños y niñas, desde el parvulario hasta su ingreso en la Universidad, mediante la formación de hábitos y la adquisición de conocimientos que ensanchasen su capacidad de acción y el horizonte de sus intereses. A la vista de la necesidad cada vez más apremiante de una comunicación internacional, y de la escolarización de sus propios hijos, Castillejo propuso una nueva creación escolar donde se dedicase especial atención al aprendizaje de las lenguas modernas.

Un objetivo primordial de esta Escuela era el ensayo, no intentado hasta entonces, de una preparación de los niños para la vida internacional, efectuada desde la infancia, paralelamente con la educación nacional, en la proporción y con los métodos adecuados a cada edad. Sir Michael C. Sadler (1937) señala en el prólogo a la obra de Castillejo Guerra de ideas en España que la «Escuela internacional Española (cuyo nombre es significativo) [...] llegó a ser una de las empresas educativas más interesantes de Europa.»

En la Memoria de la mencionada escuela (1931: 3) se afirma que «el hombre necesita una lengua privilegiada e íntima como órgano de su pensamiento y de su cultura nacional; pero será inservible para el cosmopolitismo de nuestra época si no se nutre además con el contacto inmediato de otras civilizaciones. [...] Por eso [la Escuela Internacional Española] ha introducido la enseñanza plurilingüe, dada en todos los grados por profesorado español, francés, inglés y alemán.»

Las fuerzas conservadoras publican un artículo en el periódico El Debate donde se critica la enseñanza plurilingüe a edades tan tempranas, ya que de este modo se compromete el espíritu nacional. Como respuesta, Pedro Salinas, presidente de la Asociación para la enseñanza plurilingüe, publica un artículo titulado La enseñanza plurilingüe en el periódico

\footnotetext{
${ }^{11}$ La cursiva es de la autora.

12 Vid. Trend (1921: 26).
} 
progresista El Sol de 21 de noviembre de 1928, donde defiende los postulados básicos de dicha asociación:

Más grave es la profecía de El Debate sobre los resultados de la enseñanza de las lenguas vivas a los niños entre tres y diez años. Nos dice que comprometemos con ello «el alma y el españolismo de unos cuantos muchachos» que «no sabrán jamás español» y serán «un plantel de [sic] déracinés.» Es verdaderamente temible que todo eso pueda ocurrir simplemente porque se estudian las lenguas antes de los once años. [...] creemos sinceramente que la enseñanza de las lenguas modernas, sea antes de los once años o después, lejos de ser una idea pasada de moda, contribuye eficacísimamente a la formación de espíritus amplios y tolerantes, que, sin perder ninguna de sus virtudes nacionales, sepan asimilar la universalidad cultural que la vida moderna nos impone a todos.

El número máximo de alumnos por aula era de 15. Se aprovechaban todas las actividades que realizaba el niño para ejercitarlo en el uso de las lenguas. La gramática de las lenguas extranjeras se estudiaba más tarde, cuando el niño, convertido en adolescente, ya las había adquirido como la materna «en la proporción cuantitativa que permite cada edad, primero al oído y luego por la lectura y la escritura. ${ }^{13}$ Los niños recibían las enseñanzas desde los tres o cuatro años de edad en tres idiomas: español, francés e inglés o alemán, dedicando a cada idioma un tercio de la sesión escolar. Entre los diez y los doce años comenzaban el estudio del cuarto idioma. Las lenguas muertas, que sólo pueden ser estudiadas gramaticalmente, se dejaban para los últimos años del Bachillerato. Según consta en la Memoria (1931: 5), «En los tres cursos que la Escuela ha hecho se ha apreciado la prodigiosa facilidad, en las edades de tres a nueve años, para asimilar las lenguas, como por adivinación, y referidas a las personas de quienes se escuchan. De aquí la exigencia inexorable de que cada maestra no use jamás otra que la suya.» ${ }^{14}$ Estas lenguas no se aprendían como materias de estudio, sino como medios de comunicación por el uso, lo mismo que la lengua materna en el hogar. Es interesante observar el germen de la moderna distinción que Widdowson (1978) estableció entre los conceptos de usage y use. $^{15}$ "Entre los tres y los cinco años el niño educa su oído, atesorando palabras, que a veces, no comprende, a las cuales va atribuyendo después con el uso, un significado cada vez más preciso.» ${ }^{16}$ Según consta en dicha Memoria, al cumplir los diez u once años, los primeros alumnos de la Escuela Internacional Española, aunque ingresados tardíamente y sin haber pasado en ella más que tres cursos, han adquirido conciencia de los caracteres diferenciales de cada lengua, han comenzado a derivar hacia la lógica gramatical, leen y entienden, han iniciado los ejercicios de composición y hasta reciben ya en los idiomas extranjeros lecciones elementales de geografía, ciencias naturales, narraciones históricas y cuentos. ${ }^{17}$ Pero el punto esencial que se determinó con este experimento fue que contra lo que se había previsto, se ha comprobado que no existe retraso alguno y que los niños

\footnotetext{
${ }^{13}$ Vid. Memoria de la escuela internacional española. Madrid. Curso 1929-1930.

${ }^{14}$ Esta norma se observó muy estrictamente. Castillejo separó del centro a una profesora por hablar español en clase de francés, según carta de Castillejo a Pedro González Bueno, 9-12-1932.

${ }^{15}$ Cf. H.G. Widdowson (1978: 18-19): «I have suggested that a distinction might be made between language usage and use. The first of these is the citation of words and sentences as manifestations of the language system, and the second is the way the system is realized for normal communicative purposes. [...] A knowledge of use must of necessity include a knowledge of usage but the reverse is not the case.

${ }^{16}$ Vid. Memoria de la escuela internacional española.. Madrid. Curso 1929-1930.

${ }^{17}$ Ibidem.
} 
se hallan al nivel de los de igual edad en otras escuelas. ${ }^{18}$ En este sentido, Castillejo señala en el periódico El Sol (29-9-1932):

\begin{abstract}
El ensayo iniciado hace cuatro años en la Escuela Internacional Española de Madrid era cosa enteramente nueva, pero [...] no envolvía riesgo. Se trataba sencillamente de poner al lado de las enseñanzas ordinarias en castellano una serie de enseñanzas dadas por profesorado extranjero en francés, inglés y alemán, o sea la alianza de cuatro grandes escuelas representando cuatro grandes civilizaciones, para dar al niño desde su infancia hábitos y conocimientos que van a serle indispensables en la moderna vida cosmopolita.

Podían prever el éxito. Los niños al llegar a los doce años, manejan ya tres lenguas, que continuarán usando a diario hasta llegar a la universidad. [...] Pero la fecundidad de los experimentos suele venir de hallazgos imprevistos. Y aquí lo imprevisto ha sido que no se aprecia retraso alguno en niños a quienes, desde los cuatro a los doce años, se tiene a media ración de escuela española. Hemos hallado pues, dos tesoros: unos años de niñez disponibles y una mentalidad infantil más rica y flexible de los que sospechábamos.
\end{abstract}

También existía como actividad complementaria de la Escuela el club internacional infantil, que en sesiones de tarde de tres a cinco organizaba actividades rítmicas, deportivas, manuales, musicales y dramatizaciones. Estas actividades eran dirigidas por las profesoras en sus varias lenguas. Se organizaban además clases especiales de lenguas vivas para niños y niñas mayores de ocho años que no siguiesen los cursos regulares de la Escuela o que necesitasen atención especial.

\title{
3. Metodología y materiales didácticos
}

En las directrices metodológicas que figuran en la memoria se afirma que «los idiomas extranjeros se aprenden mediante el insistente uso cotidiano en todas las actividades del niño, aprovechando la edad en que su oído e instinto se prestan más a ello. Las maestras extranjeras no hablan en la escuela otra lengua más que la suya. Se valen de juguetes, siluetas recortadas, grabados, estampas, tarjetas postales, revistas ilustradas, carteles anunciadores y de cuanto material intuitivo pueden reunir para que cada palabra llegue a los niños asociada al objeto o acción que representa. Como podemos observar, se cumple aquí textualmente el artículo $5^{\circ}$ de los estatutos de la Asociación fonética Internacional, ligada íntimamente a la Metodología Natural o Directa, que establece «las palabras y las acciones deben enseñarse y aprenderse directamente ligadas con los objetos u acciones que representan.»

Con los alumnos más pequeños de la escuela Internacional Española se dejaba un margen mayor a la espontaneidad; la maestra subordinaba sus planes a su interés y participación. El canto, el dibujo, la recitación con mímica, los trabajos manuales, los juegos que exigen el uso de palabras o frases sucesivamente renovadas, las historias representadas con muñecos, alternaban para mantener alerta la atención y ejercitar los idiomas.

En cuanto al vocabulario, éste comienza con los nombres de objetos y ocupaciones que rodean al niño en la escuela, en el hogar, en el campo y en la calle, colores y formas, verbos

\footnotetext{
${ }^{18}$ Ibidem.
} 
de acciones que maestra y alumnos reproducen y escenas de las láminas. Se utilizan juegos con material intuitivo, como la lotería de objetos, el mercado, el ferrocarril, las muñecas; juegos acompañados de frases; juegos que suscitan una construcción gramatical correcta o el recuerdo de palabras; dibujo, recortado y plegado de papel. Y se repiten rimas y canciones infantiles, algunas dramatizadas: Frère Jacques, Les canards, Marie trempeton apin [sic], Battons le fer, Sur le pont d'Avignon, Muh, Muh, Muh, so spricht die bunte kuh, Jack and Jill, Little Jack Horner, I saw a little bird, Sing a song of sixpence, entre otras.

Con el grupo de alumnos un poco mayores el vocabulario se amplía con descripciones y acciones, se introduce el diálogo, la construcción de frases, los ejercicios de comparación y oposición, la variación de formas verbales, la lectura y escritura sencillas, y el hallazgo de palabras suprimidas en una oración. Por último, con el grupo de alumnos más avanzados se llega, en inglés y en francés, a comenzar la lectura de libros de cuentos y la redacción de resúmenes, y a exponerles oralmente la historia de los medios de comunicación, del traje, de los cultivos, etc.

Como se deduce de las actividades descritas podemos encontrar claros antecedentes de actividades utilizadas en la actualidad, tales como role play, listening activities, TPR (Total Physical Response, communication games, information gap activities, la enseñanza de otros contenidos a través del inglés, francés y alemán etc. Hemos encontrado en el Archivo del Museo Pedagógico de Madrid (caja sin catalogar) materiales para la enseñanza del inglés a los niños mediante pequeñas obras teatrales. Se intenta además enseñar al niño algún aspecto interesante como por ejemplo la seguridad vial, o a escapar de algún tipo de peligro al que pueden estar expuestos y al mismo tiempo practicar una lengua extranjera. Pasamos a transcribir algún ejemplo de estas pequeñas obras de teatro: ${ }^{19}$

\section{Safety in Mother Goose Land \\ (Slightly abridged) \\ Characters}

\begin{tabular}{|l|r|}
\hline Mother Goose & Old mother Hubbard and Dog \\
\hline King Safety & Tom, the piper's son \\
\hline Little boy blue & Simple Simon \\
\hline Boo Peep & Johnny Green \\
\hline Jack and Jill & Johnny Stout \\
\hline Old woman who lived in a shoe & Dr. Foster \\
\hline Doctor & Nurse \\
\hline
\end{tabular}

\footnotetext{
${ }^{19}$ La selección de estos materiales novedosos muestra un cambio radical con respecto a los materiales morales, virtuosos y religiosos que predominaban en el siglo XIX. Las historias de Mother Goose o Nursery Rhymes pertenecen a la literatura infantil de los países nórdicos basada en la imaginación y la fantasía. Los países mediterráneos, por otra parte, muestran mayor obediencia a la autoridad religiosa y prefieren como materiales historias morales, virtuosas o bíblicas condenando, en cierto modo, la fantasía y la imaginación. Como señala (Hazardd, 1946: 35) «For the Latins, children have never been anything but future men. The Nordics have understood better this truer truth, that men are only grown-up children.»
} 
(Costumes conforming to Mother goose illustrations may be made of crepe paper or cheesecloth)

Mother Goose ${ }^{20}$, personaje central de los famosos cuentos del mismo nombre, clásico de la literatura infantil norteamericana, es también el personaje central de esta obra de teatro que, junto con King Safety, da innumerables consejos a los niños sobre seguridad vial. Cada personaje entra en escena quejándose de algún peligro que le acecha, entonces Mother Goose y King Safety ofrecen su sabiduría a los niños para evitar dichos peligros, y si es necesario, $D r$. Foster y Nurse los atienden y curan sus heridas:

Mother Goose: Now, what has happened to little Bo-Peep who always so carefully tends her sheep?

Boo-Peep: (Tearfully) I ran out from behind a parked truck to find the tails for my sheep and an automobile knocked me over.

Mother Goose: It is a lucky thing you were not killed [...]

Las siguientes obras de teatro tienen también el mismo tono didáctico de la anterior. Se trata de prevenir a los niños contra ciertos peligros:

\section{The Bunnies Adventure}

This little play is designed to teach children how young rabbits have to learn the lessons which will make them able to escape from their enemies, just as children have to learn the lessons that will safeguard them from the dangers to which they will be exposed all their life.

Characters:

Mother Rabbit

The little bunnies: Bob-tail, Brownie, Scamp.

Long legs, a hare

Buck, the farmer's dog

Farmer Adams

Otra de las obras utilizadas para las actividades de teatro en inglés ${ }^{21}$, siguiendo la misma línea didáctica, tenía el siguiente título:

\section{The Fairies hold a Safety Court}

A play warning children against the danger of playing with fire whether at home or in a wood and other dangers.

Place:

in a forest

Characters:

Elf King

Fairy Queen

\footnotetext{
${ }^{20}$ Mother Goose Stories is the American equivalent to Nursery Rhymes.

${ }^{21}$ El Instituto Internacional de Madrid, llamado también Instituto de Boston colabora con la ILE (Institución Libre de Enseñanza) enviando profesoras norteamericanas para las clases y actividades en inglés.
} 
Dwarf

Boys

Girls

En la sección de párvulos, como en la elemental, la enseñanza de las lenguas extranjeras se basaba en: a) el método Directo (o Natural), con auxilio de juguetes, siluetas, carteles murales, libros ilustrados, visitas a museos o a cualquier lugar que ofreciese objetos y escenas capaces de provocar el diálogo y enriquecer el vocabulario; b) canciones, dramatizaciones, recitaciones; c) las actividades manuales (arts and crafts) que podían suscitar más el uso del lenguaje; d) ejercicios de lectura que comenzaban a realizar los niños entre los ocho y los nueve años de edad.

Como hemos podido observar, la aproximación intuitiva a la enseñanza de las lenguas vivas y las actividades y materiales utilizados en la Escuela Internacional Española llegan a sorprender al lector moderno por su actualidad. Muchos de estos materiales fueron diseñados por las becarias de los intercambios promovidos por la JAE como hemos mencionada más arriba.

Eran muchas las actividades organizadas dentro del marco general de la ILE para promover el estudio de las lenguas y culturas extranjeras. Sin embargo, no debemos de perder de vista el hecho de que en ese momento sólo una pequeña porción de la sociedad, los intelectuales y clases acomodadas se beneficiaban de tales iniciativas. El interrogante que queda abierto es, por lo tanto, qué hubiese ocurrido si tales actividades no se hubiesen visto truncadas por el comienzo de la guerra civil y se hubiesen ido generalizando a toda la población escolar.

\section{Conclusión}

Esta actitud aperturista hacia Europa, en especial hacia Inglaterra, a la cual Castillejo llamaba maestra de vida, era considerada peligrosa y extranjerizante por los conservadores. Los principios que más admiraba Castillejo de la educación inglesa eran según sus propias palabras los siguientes:

La libertad es el supuesto de toda acción educadora. Solamente dentro de ella puede formarse el carácter mediante el poder de iniciativa y la adquisición de hábitos [...] La libertad no significa ausencia de coacción, sino ausencia de coacción arbitraria impuesta por el educador.

La única represión verdaderamente eficaz ha de proceder de la acción misma del educando, [...] Así se llega a adquirir el poder de refrenar los deseos y apetitos que la razón no autoriza: la educación es fundamentalmente potencia de inhibición. En la tradición de las escuelas inglesas está el fomento de la espontaneidad, con supresión de todo sistema de desconfianza y espionaje; consideran que tratando a un niño como honrado, leal y caballero, se contribuye eficazmente a que lo sea. ${ }^{22}$

\footnotetext{
${ }^{22}$ J. Castillejo «Las Bases de la Educación Inglesa» BILE. 1918. Vol. XLII, pp. 166-177.
} 
En este sentido, Castillejo denuncia la situación predominante en las escuelas religiosas:

La educación inglesa representa la antítesis del ideal monástico [...] y de los métodos dominantes hoy todavía en las escuelas de los Jesuitas. Hay en el fondo de esos métodos un concepto pesimista del hombre, y a ese concepto corresponde, como misión primordial del educador, una intervención constante para suprimir tropiezos e influjos perniciosos, y una vigilancia incesante del niño, porque sólo la presencia y la presión de la autoridad pueden mantener en jaque los innatos, perversos instintos. ${ }^{23}$

Castillejo hizo todo lo posible para introducir tanto la metodología como los ideales educativos ingleses en las escuelas españolas, ya que el sistema inglés se oponía totalmente al principio autoritario y a las ideas medievales que aún se sostenían en el sistema educativo español. La Institución Libre de Enseñanza estuvo desde el principio relativamente libre de la inspección y del control tanto del gobierno como de la iglesia y por este motivo prosperó aceleradamente. Tomó partido por el individualismo, la libertad de pensamiento y de todo tipo de autoridad.

No es extraño que estas ideas tan progresistas para la época causaran recelo en buena parte de la sociedad, especialmente entre los vencedores de la Guerra Civil. Es así como en el discurso del primer ministro de Educación Nacional del régimen, Pedro Sainz Rodríguez, pronunciado en Pamplona el 30 de junio de 1938, se dice:

Frente a ese dogma del naturalismo hedonista que afirma que el hombre es naturalmente bueno, hay que contraponer la doctrina católica de que el hombre es malo por causa del pecado original y de la caída que envileció su alma.

Como señala (Pérez Galán, 1976: 17-19) «La pedagogía revolucionaria ha consistido además en borrar la idea de Patria como entidad moral de la conciencia de los españoles.» Con respecto a estos temas, José Pemartín, Director General de Enseñanza Media bajo los ministerios de Sainz Rodríguez y comienzos del de Ibáñez Martín, señalaba en su libro Qué es lo Nuevo que hay que devolver a la enseñanza oficial su fondo católico y patriótico del que la despojaron «las generaciones de estudiantes y catedráticos anti-españoles, de orientación exótica, irreligiosa, masónica, que desde la ciudadela de la Institución Libre de Enseñanza caciqueaban a su gusto en toda la enseñanza Oficial.»

Por decreto del 17 de mayo de 1949 se dispone la incautación de los bienes de la Institución Libre de Enseñanza, entre ellos, de la Escuela Internacional Española. Castillejo se exilia en Inglaterra hasta su muerte. Dichos bienes pasaron a estar adscritos al Ministerio de Educación Nacional. a este tema Refiriéndose a este tema Pérez Galán, 1976: 19, afirma «Así se acaba con una Institución que durante más de sesenta años acrecentó como ninguna otra, el acervo cultural de nuestra Patria [...] La historia ha venido a quitar la razón a quienes se ensañaron, tras la guerra civil, con los que siempre predicaron y ejercieron comprensión, tolerancia y respeto a la conciencia ajena.»

También hemos podido comprobar que los principios educativos y metodológicos sustentados en la actualidad contienen componentes históricos, que han ido evolucionando a través

\footnotetext{
${ }^{23}$ Ibidem.
} 
del tiempo, y que continuarán evolucionando en el futuro. La visión histórica despierta, pues, un espíritu crítico en el docente que a su vez sirve de motivación para la innovación y el cambio. Al mismo tiempo evitamos que una serie de ideas, teorías, conocimientos, prácticas y técnicas que se han acumulado con el correr de los siglos se pierdan; en consecuencia, las generaciones siguientes no tendrán que redescubrirlas o reinventarlas, sino que se añadirán al cuerpo de conocimientos que conforman la disciplina de la enseñanza-aprendizaje de lenguas extranjeras. ${ }^{24} \mathrm{~A}$ menudo nuestra ignorancia del pasado nos lleva a volver a revivir viejos conflictos como algo moderno y actual justamente por falta de perspectiva histórica. Muchas ideas que en la actualidad se consideran revolucionarias corresponden, en realidad, a nuevas etiquetas o nuevos planteamientos de ideas y aproximaciones de siglos anteriores. En donde sí ha habido una gran evolución es en los medios y en la calidad de los materiales aunque la filosofía predominante no sea totalmente novedosa. Entendemos que resulta peligroso analizar hechos pasados con parámetros actuales y por lo tanto, lo único que podemos comprobar objetivamente es la existencia de la Institución Libre de Enseñanza y su novedosa aproximación metodológica en el estudio de lenguas extranjeras.

De este modo, hemos pasado revista, dentro del ámbito de la ILE, a los antecedentes de ideas y aproximaciones gloso-didácticas muy populares en la actualidad, tales como la enseñanza comunicativa de los idiomas modernos, la enseñanza del inglés a través del inglés (presupuesto de la metodología Directa o Natural), el aprendizaje centrado en el alumno, la introducción de la lengua extranjera desde la escuela infantil a través de actividades lúdicas, la enseñanza de otras materias a través de las lenguas extranjeras, entre otras. La guerra civil supuso un prolongado paréntesis en la actividad pedagógica renovadora propiciada por la ILE, que prácticamente no volvió a resurgir hasta el último tercio del siglo XX.

\section{REFERENCIAS BIBLIOGRÁFICAS}

Bases y Estatutos de la Institución Libre de Enseñanza. Madrid. 1876.

Castillejo, J. «Las Bases de la Educación Inglesa» Boletín de la Institución Libre de Enseñanza.1918. Vol.XLII. pp. 166-177.

Castillejo, J. «La Enseñanza de las Lenguas Vivas» El Sol, 30-12-1928.

Castillejo, J. «La Enseñanza Plurilingüe» El Sol, 1-1-1929.

Castillejo, J. «¿Malgastamos la niñez de nuestros hijos?» El Sol, 29-9-1932.

Castillejo, J. (1937). Ed. de (1976). Guerra de ideas en España. Madrid: Ediciones de la Revista de Occidente.

Colección Legislativa de España. Tomo XXVIII, vol.1º de 1907, pp. 49-57.

Gamero Merino, C. (1987). «La Escuela Internacional Española.» Actas de las segundas jornadas de educación: Segundas Jornadas de Educación. José Castillejo y la Política Europeísta para la reforma educativa española. 221-238. Diputación de Ciudad Real. Área de cultura. Hazard, P. (1946). El pensamiento europeo en el siglo XVIII. Madrid: Imp. De Viuda de Galo Saez. Howatt, A.P.R (1983). «Five Hundred Years of English Language Teaching.» English Language Teaching Journal 37: 262-265.

Howatt, A.P.R. (1984). A History of English Language Teaching. Oxford: University Press.

Howatt, A.P.R. (1984). «Language Teaching Traditions: 1884 Revisited.» English Language Teaching Journal 38: 279-282.

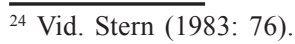


Jespersen, O. (1925). «La enseñanza de las lenguas extranjeras» Boletín de la Institución Libre de Enseñanza,, t. XLIX, pp. 358-370.

Kelly, L.G. (1969). 25 Centuries of Language Teaching. Rowley, Massachusetts: Newbury House. Krashen, S.D. \& Terrel, T.D. (1983). The Natural Approach. Exeter: Prentice Hall International.

Memoria de la Escuela Internacional Española. Madrid. Curso 1929-1930.

Memoria de la Escuela Internacional Española. Madrid. Curso 1931-1932.

Pérez Galán, M. «La ILE en los comienzos del Franquismo» Cuadernos de Pedagogía. Vol. 22, pp. 17-19.

Stern, H.H. (1983). Fundamental Concepts of Language Teaching .Oxford: University Press.

Trend, J.B. (1921). A Picture of Modern Spain. Men \& Music. London: Constable and Company Limited.

Viña Rouco, M ${ }^{\mathrm{a}}$ M. (2000). La enseñanza de las lenguas vivas en España (1800-1936), con especial referencia a la lengua inglesa. Tesis Doctoral. USC.

Widdowson, H.G. (1978). Teaching Language as Communication. Oxford: University Press. 